\title{
Dust and the art of Galactic map making
}

\author{
Douglas J. Marshall ${ }^{1}$, Gilles Joncas ${ }^{1}$, Anthony P. Jones ${ }^{2}$, \\ Annie C. Robin ${ }^{3}$, Céline Reylé ${ }^{3}$ and Mathias Schultheis ${ }^{3}$ \\ ${ }^{1}$ Département de physique, de génie physique et d'optique et Centre de recherche \\ en astrophysique du Québec, Université Laval, Québec, QC, G1V 0A6, Canada \\ email: douglas.marshall.1@ulaval.ca \\ ${ }^{2}$ Institut d'Astrophysique Spatiale, bâtiment 121, Université Paris-XI, Orsay, 91405, France \\ ${ }^{3}$ Observatoire de Besançon, Institut UTINAM, Université de Franche-Comté, \\ BP 1615, 25010 Besançon Cedex, France
}

\begin{abstract}
A Galactic model of stellar population synthesis is used along with a genetic algorithm to reconstruct the three dimensional dust distribution in the Milky Way. We have applied this technique towards over 1500 IRDC cloud candidates, for which we recovered distances and masses for 1259 of them. Aside from giving us the distance to the dust, the three dimensional extinction map also provides us with a temperature independent measure of its density. This new method is independent of any kinematical information, thus providing a new way to obtain information on the Galactic distribution of the ISM. It is a good complement to existing measures which are solely based on molecular gas kinematics as both methods are completely independent and both are affected by different systematics. It will be able to provide valuable distance information for use in the analysis and interpretation of far-infrared and sub-millimetre observations by Herschel and Planck. In the future it could be used with deeper stellar observations or observations at longer wavelengths in order to probe even higher density clouds and to even larger distances.
\end{abstract}

Keywords. ISM: clouds - dust, extinction - ISM: structure - Galaxy: structure

A three dimensional map of the dust distribution in the Milky Way has a number of uses. Apart from providing us with an alternate view on the structure of our home Galaxy, it can be put to many uses in order to gain a better understanding of the physical conditions in the ISM as well as helping us to understand the evolution of our Galaxy. In order to produce such a map, the Besançon model of Robin et al. (2003) is used along with a genetic algorithm as implemented by Charbonneau (1995). We have applied this new three dimensional extinction technique towards over 1500 IRDC cloud candidates from Simon et al. (2006a), for which we recovered distances and masses for 1259 of them, including over 1000 previously unmeasured clouds. The spatial distribution of the clouds is found to be concentrated in the molecular ring and along the spiral arms. The resulting three dimensional dust distribution can be used to help separate the dust emission signal that will be observed by Herschel and Planck into discrete components along the line of sight. In the future the $3 \mathrm{~d}$ extinction method will be used with deeper stellar observations or observations at longer wavelengths in order to probe even higher density clouds and to even larger distances.

\section{References}

Charbonneau, P. 1995, ApJS, 101, 309

Robin, A. C., Reylé, C., Derrière, S., \& Picaud, S. 2003, A\&A, 409, 523

Simon, R., Jackson, J. M., Rathborne, J. M., \& Chambers, E. T. 2006, ApJ, 639, 227 